\title{
Peripheral Odontogenic Myxoma: A Review of the Literature and Report of Two Cases
}

\author{
Erich J. Raubenheimer • Claudia E. Noffke
}

Received: 3 December 2010/Accepted: 23 February 2011/Published online: 19 April 2011

(C) Association of Oral and Maxillofacial Surgeons of India 2011

\begin{abstract}
Two cases of peripheral odontogenic myxoma with a verifiable location in gingival soft tissue and without bone involvement were compared with those reported in the literature. This study showed that they form a distinct albeit rare clinical entity with a potential to grow into large disfiguring lesions. The probability that small peripheral odontogenic myxomas are interpreted as edematous irritation fibromas may contribute to the small number of peripheral odontogenic myxomas recorded in the literature. The differential diagnosis of soft tissue myxoid proliferations is discussed.
\end{abstract}

Keywords Odontogenic myxoma $\cdot$ Peripheral odontogenic tumor $\cdot$ Irritation fibroma

\section{Introduction}

Odontogenic myxoma $(\mathrm{OM})$ is a locally invasive neoplasm unique to the tooth bearing areas of the jaw bones. It is believed to arise from odontogenic ectomesenchyme and consists of rounded and angular cells embedded in abundant mucoid stroma [1]. OMs occur over a wide age range. In a series on odontogenic tumors in Africa, OMs were

\author{
E. J. Raubenheimer ( $\bowtie)$ \\ Oral Pathology, School of Oral Health Sciences, \\ University of Limpopo, Box D24, Medunsa Campus, \\ Medunsa 0204, \\ South Africa \\ e-mail: ejraub@ul.ac.za \\ C. E. Noffke \\ Radiology, School of Oral Health Sciences, University \\ of Limpopo, Medunsa Campus, Medunsa 0204, South Africa \\ e-mail: noffke@ul.ac.za
}

reported to be more common than odontomas [2]. Most of the recently reported large series on OM [3-5] make no mention of the peripheral variant.

Peripheral odontogenic myxoma (POM) is rare with less than 6 cases manifesting in the gingival soft tissue reported in the literature [6-9]. No data is subsequently recorded in standard Oral Pathology textbooks on their clinico-pathological features. POM is reported to present clinically as an exophytic gingival mass, the overlying epithelium is generally unaffected and no bony involvement is present [6]. POM may be difficult to differentiate microscopically from other tumors with myxoid features, many of which have malignant characteristics. Their low prevalence in gingival soft tissue contribute to POMs being generally excluded from the differential diagnoses of extra bony myxoid proliferations.

The purpose of this study was to record cases of peripheral odontogenic myxoma (POM) in a large sample of OMs previously studied in an African population sample [2].

\section{Case Reports}

Thirty cases of centrally located odontogenic myxomas $(\mathrm{OM})$ from a series of $52 \mathrm{OMs}$ diagnosed over a period of 23 years at the University of Limpopo, which serves mainly a rural and peri urban black African population, were reported in 2007 [2]. Amongst the cases excluded from this study on central OMs, were two cases of histologically verified peripheral odontogenic myxoma (POM). Both cases of POM presented as painless soft tissue masses interfering with oral function. The medical histories of both patients were unremarkable. The first patient, a 53 year old female, gave a history of an epulis that had been removed 

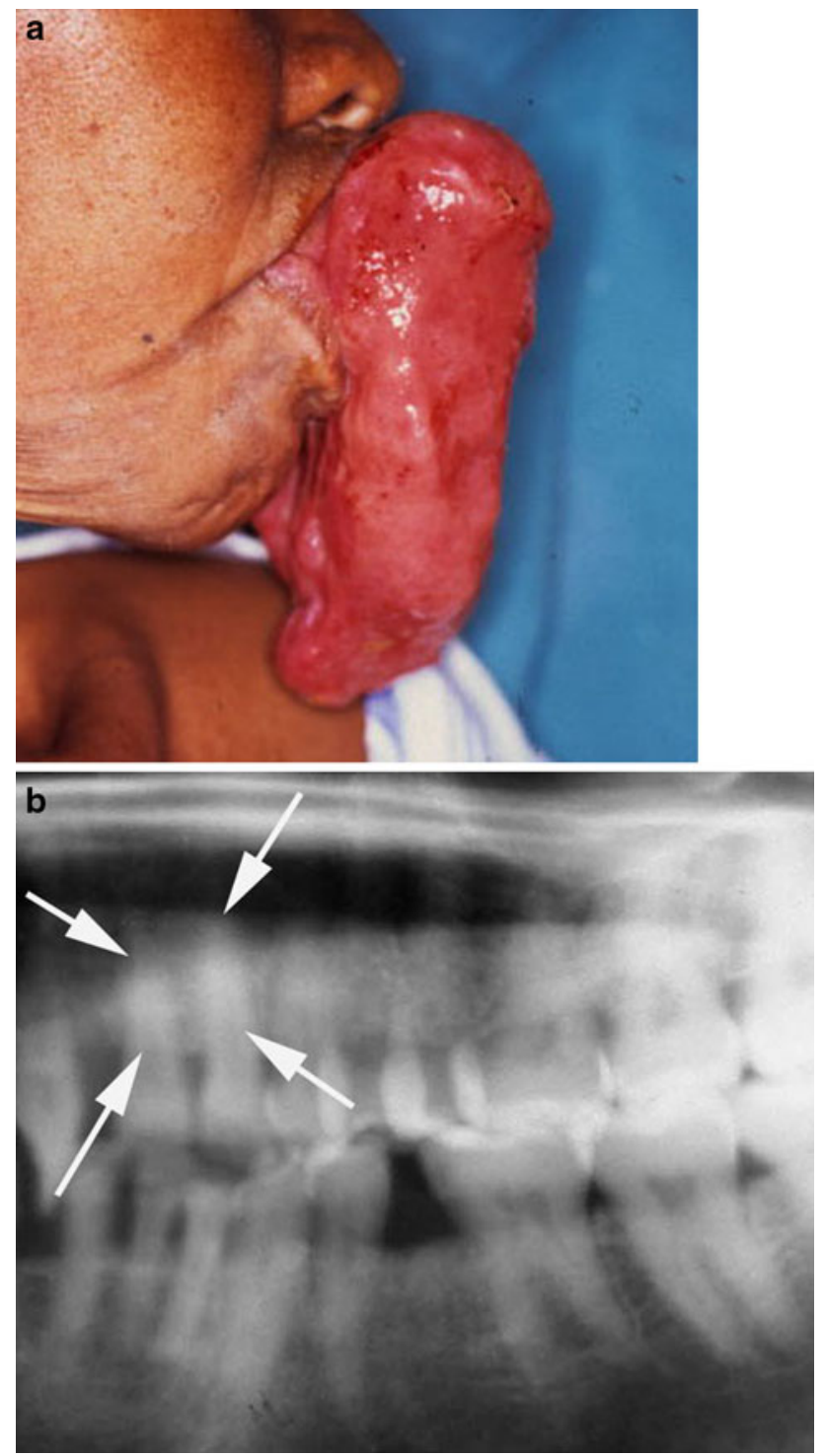

Fig. 1 Case 1: a Exophytic mass attached to the anterior maxillary gingiva and protruding from the mouth. b Cropped panoramic radiograph. The arrows indicate the site of attachment of the neoplasm to the anterior gingiva

twice at the same site 5- and 4 years previously. As far as she could recall, no microscopic report was available on the lesion. A large pedunculated tumor measuring $12 \times 6 \times 5 \mathrm{~cm}$ that protruded from the oral cavity and extended beyond the lower lip was noted (Fig. 1a). The tumor had a soft consistency and was attached with a narrow base of approximately $2 \mathrm{~cm}$ in diameter to the labial gingiva in the left central and lateral incisor area of the maxilla. Although the maxillary incisors and left canine showed extensive calculus deposits and were mobile, no bony involvement was present (Fig. 1b). The lesion was covered by atrophic epithelium with foci of ulceration and a clinical diagnosis of a low grade sarcoma was made. In order to alleviate the patients discomfort it was decided to
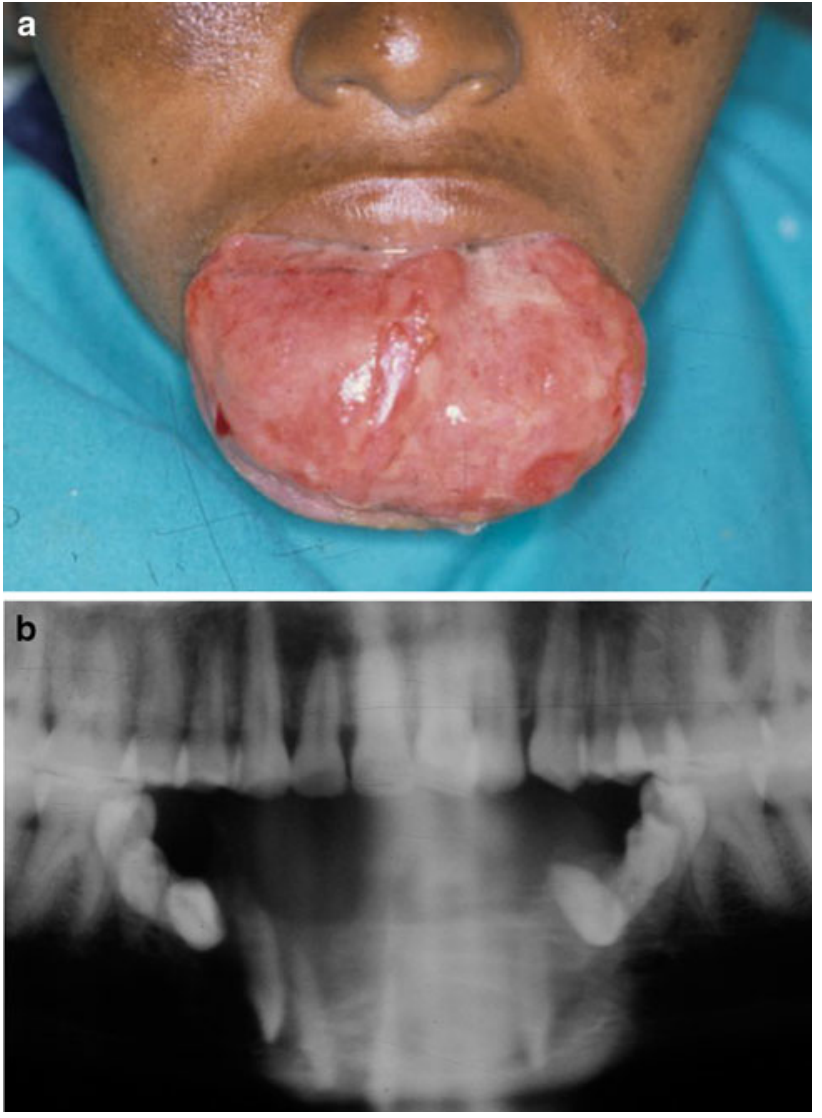

Fig. 2 Case 2: a Exophytic mass attached to the mandibular lingual gingiva. b Cropped panoramic radiograph showing displacement of the incisors, canines and premolar teeth. Note the cuffing of the alveolar bone

remove the bulk of the tumor by incising through the narrow base. Microscopic examination established a diagnosis of odontogenic myxoma. The patient was followed for the past 7 years and is tumor free.

The second patient was a 38 year old female with an erythematous and partly eroded tumor measuring $8 \times 6 \times 4 \mathrm{~cm}$ protruding from the mouth (Fig. 2a). The lower lip was stretched around the inferior border of the tumor. With intra-oral examination the tumor was found to be attached with a narrow base to the lingual gingiva in the region of the anterior mandibular teeth. The mandibular canine and incisor teeth were tilted forward in a horizontal plane and the mandibular alveolar margin showed cuffing (Fig. 2b). A surgical biopsy was diagnosed as an odontogenic myxoma. The tumor was removed surgically and lack of bone involvement with an intact mandibular periosteum was confirmed. The patient was lost for follow-up.

During the biopsy procedure of both cases the surgeons reported a tumor with a gelatinous loose structure. Microscopic examination of both cases showed a haphazard arrangement of spindle shaped and stellate shaped cells in a loosely woven stroma composed of delicate collagen 


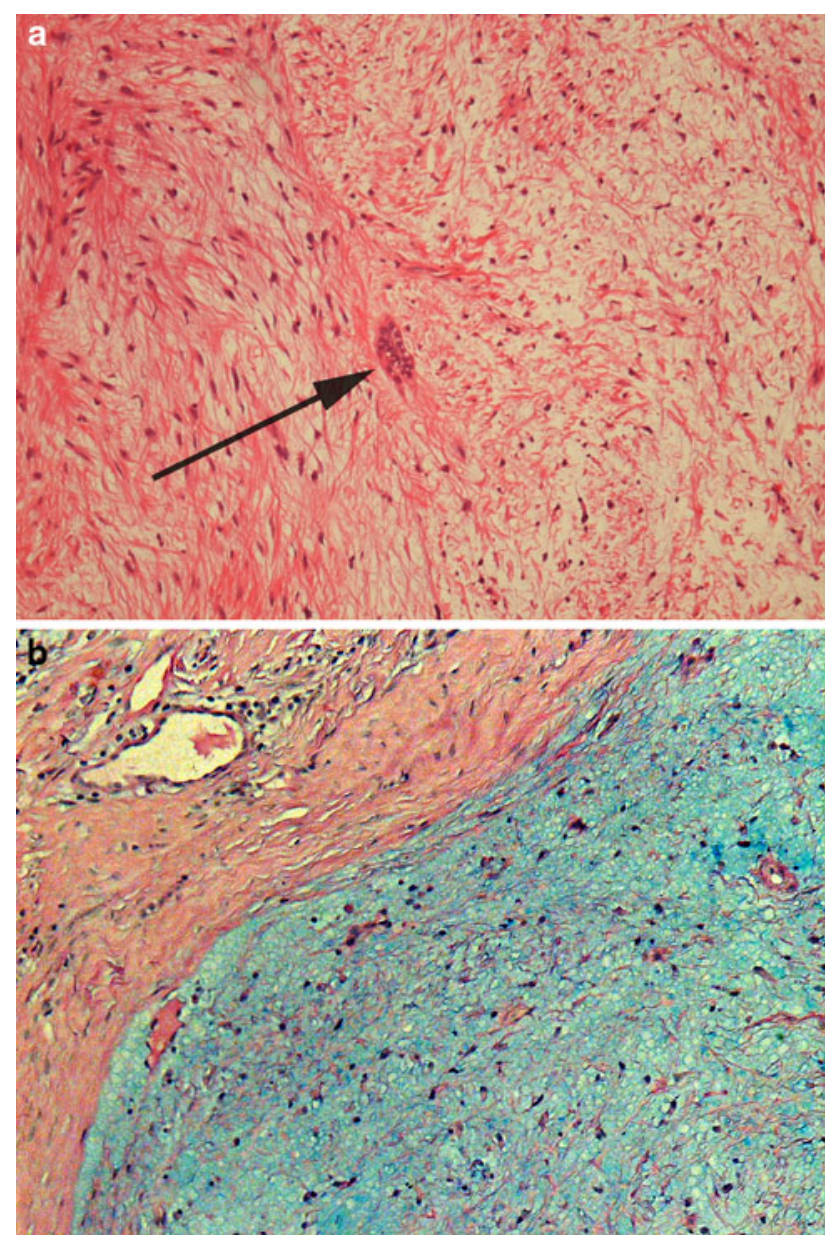

Fig. 3 a Photomicrograph of the myxoid core of the neoplasm in Case 1 showing one inactive odontogenic epithelial rest (arrow, H\&E stain $\times 200$ ). b Alcian blue-PAS stain showing the glycosaminoglycans deposits between the delicate collagen fibrils of Case 2. Note the lack of connective tissue ground substance in the normal collagen of the sub mucosa (left upper corner, $\times 200$ )

fibrils in a stroma rich in glycosaminoglycans. The cells were vimentin positive and S100 negative. An exhaustive search identified inactive odontogenic epithelial rests in both tumors (Fig. 3).

\section{Discussion}

$\mathrm{OM}$ is a locally invasive neoplasm unique to the tooth bearing areas of the jaw bones. It is believed to arise from odontogenic ectomesenchyme of the dental follicle. In the fully developed jaw, remnants of odontogenic ectomesenchyme are found in the periodontal ligament (radicular part of the tooth follicle) and gingiva (coronal part of the tooth follicle). It is therefore conceivable that ectomesenchymal cells in both these locations could potentially serve as a stem cell population for neoplastic proliferations with the microscopic features of an OM. Although OM is generally considered to be a rare odontogenic neoplasm, they appear to be more frequent in series reported in Africa than in other parts of the world [2,4]. OMs are more common in females and occur over a wide age range with an average age at presentation of 31.3 years [2]. By far the most common type of $\mathrm{OM}$ is the central variety which develops from odontogenic ectomesenchyme located within the periodontal ligament space. The reported incidences of POM are significantly below those of other peripheral odontogenic tumors like the peripheral ameloblastoma (1\% of all ameloblastomas [10]), peripheral odontogenic fibroma (total number of reported cases is over 150 [10]) and even the rare peripheral dentinogenic ghost cell tumor [11]. No information is subsequently recorded on POMs in standard textbooks of Oral Pathology. In general peripheral odontogenic neoplasms and most notably peripheral ameloblastomas are less aggressive than their central counterparts [11]. Although this is supported for POM by some authors [9], according to our experience this does not seem to apply to all POMs as one of our patients gave a history of several recurrences despite local excision.

Only four case reports of POM appear in the English literature [6-9]. In one of the reports, the authors use the term "soft tissue myxoma" for the gingival mass showing the microscopic features characteristic for OM [6]. We propose application of the term POM to this tumor as "soft tissue myxoma" may lead to confusion with the other non odontogenic myxomatous proliferations found in soft tissue. The two cases reported in our paper measure 9- and $10 \mathrm{~cm}$ in largest dimension respectively and are the largest examples of POM reported in the literature. Their sizes are indicative of their unlimited growth potential if left untreated, a phenomenon which distinguishes POM from reactive non-neoplastic polypoid gingival growths such as irritation fibromas.

The microscopic assessment of a poorly circumscribed myxoid proliferation outside bone is complex. The most likely diagnoses of these proliferations in the oral cavity include a myxoid area in a pleomorphic adenoma [12], myxoid change in a fibrosarcoma or plexiform neurofibroma, myxoid liposarcoma, botroid type embryonal rhabdomyosarcoma, myxoid type chondrosarcoma, nerve sheath myxoma and chondromyxoid fibroma [13]. Microscopic examination of a representative biopsy is important for the accurate diagnosis of myxoid soft tissue neoplasms in any location. OMs show little encapsulation and the growth may be quite rapid due to the accumulation of mucoid ground substance, thereby mimicking an aggressive neoplasm [1]. The neoplasm is composed of haphazardly arranged stellate, spindle shaped and round cells in a loose myxoid stroma. Although the nuclei of the cells may show atypical features, mitoses are rarely seen. The production of abundant myxoid ground substance that stains positive for glycosaminoglycans is the histochemical 
hallmark of the neoplasm. Although inactive odontogenic epithelial remnants are not required for the diagnosis [11], they were identified in both our cases. Most small POMs are likely to be diagnosed clinically and microscopically as irritation fibromas (or fibrous epulis) unless conspicuous groups of inactive odontogenic epithelium, the typical delicate vascular network and stellate fibroblasts alert the Pathologist to a diagnosis of POM. Irritation fibromas are frequently inflamed and subsequent edematous change could mimic the typical myxoid appearance of POM. The true incidence of POMs may therefore be higher than reported. Histochemical stains for connective tissue ground substance could be helpful in distinguishing edematous change from the myxoid appearance of connective tissue ground substance deposits in POMs. The collagen content may be variable and a delicate capillary network is present. Scattered islands of inactive odontogenic epithelium, often surrounded by a zone of hyalinization, could be present in the minority of OMs $[1,14]$. Immunohistochemistry is of limited value in establishing a final diagnosis as the neoplastic cells share antigenic characteristics with many of the non odontogenic myxoid proliferations. Although positive staining with antibodies directed against vimentin and muscle specific actin have been reported [11], a specific marker for cells of dental ectomesenchymal origin is lacking.

\section{References}

1. Kramer IRH, Pindborg JJ, Shear M (1991) Histological typing of odontogenic tumours, 2nd edn. Springer Verlag, Berlin, p 23
2. Noffke CEE, Raubenheimer EJ, Chabikuli NJ, Bouckaert MR (2007) Odontogenic myxoma: review of the literature and report of 30 cases from South Africa. Oral Surg Oral Med Oral Radiol Endod 104(1):101-109

3. Kaffe I, Naor H, Buchner A (1997) Clinical and radiological features of odontogenic myxomas of the jaws. Dentomaxillofac Radiol 26:299-303

4. Ladeinde AL, Ajayi OF, Ogunlewe MO, Adeyemo WL, Arotiba GT, Bangbose BO (2005) Odontogenic tumors: a review of 319 cases in a Nigerian teaching hospital. Oral Surg Oral Med Oral Radiol Endod 99:191-195

5. Lo Muzio L, Nocini P, Favia G, Procaccini M, Mignogna MD (1996) Odontogenic myxoma of the jaws: a clinical, radiologic, immunohistochemical and ultrastructural study. Oral Surg Oral Med Oral Radiol Endod 82(4):426-433

6. Perrotti V, Rubini C, Fioroni M, Piatelli A (2006) Soft tissue myxoma: report of an unusual case located on the gingiva. J Clin Periodontol 33(1):76-78

7. Ramaraj PN, Shah SP (2003) Peripheral myxoma of the maxilla. A case report. Indian J Dent Res 14(1):67-69

8. Shimoyama T, Horie N, Kato T, Tojo T, Nasu D, Kaneko T, Ide F (2000) Soft tissue myxoma of the gingiva: report of a case and review of the literature of soft tissue myxoma in the oral region. J Oral Sci 42(2):107-109

9. Aytac-Yazicioglu D, Eren H, Görgún S (2008) Peripheral odontogenic myxoma located on the maxillary gingiva: a report of a case and review of the literature. Oral Maxillofac Surg 12(3):167-171

10. Neville BW, Damm DD, Allen CM, Bouquot JE (2002) Oral and maxillofacial pathology, 2nd edn. Saunders, China, pp 618-637

11. Irzzi G, Rubini C, Fiorini M, Piatelli A (2007) Peripheral dentinogenic ghost cell tumor of the gingiva. J Periodontol 78(8): $1635-1638$

12. Seifert G (ed) (1991) Histological typing of salivary gland tumours. Springer-Verlag, Berlin, pp 11-12

13. Enzinger FM, Weiss SW (1988) Soft tissue tumors, 2nd edn. C.V. Mosby Company, St. Louis, pp 201-296

14. Kangur TT, Dahlin DC, Turlington EG (1975) Myxomatous tumors of the jaws. J Oral Surg 33:523-528 